\title{
FORMAS DE OCORRÊNCIA DE ALUMÍNIO E FÓSFORO EM MINÉRIOS DE FERRO
}

\author{
Marcela Luciana Figueirêdo Couto ${ }^{1}$ \\ Geraldo Magela da Costa ${ }^{2}$ \\ Ana Cláudia Carioca ${ }^{3}$ \\ Flávio de Castro Dutra ${ }^{4}$
}

\section{Resumo}

É bem conhecido o efeito indesejável que impurezas como o fósforo e alumínio causam aos produtos e operações de redução de minério de ferro e refino dos aços. A presença de fósforo em teores elevados torna o aço quebradiço e com fraturas. O presente estudo é realizado com o objetivo de se estudar a forma de ocorrência de alumínio e fósforo em oito tipos de minérios de ferro provenientes de minas da Vale. Extrações sequenciais visando a remoção de formas específicas do fósforo foram realizadas. Os resíduos dessas extrações e das amostras originais foram caracterizados por difração de raios $X$, espectroscopia Mössbauer e análises químicas. Os resultados das extrações químicas sugerem que $o$ fósforo e o alumínio estão associados aos óxidos de ferro. Observa-se uma correlação nítida entre a presença de fósforo e do mineral goethita para a maioria das amostras. Entretanto, algumas amostras não contêm goethita, mas possuem um teor elevado de fósforo. A associação do alumínio aos óxidos de ferro sugere que talvez não seja possível removê-lo totalmente por métodos físicos no beneficiamento.

Palavras-chave: Minério de ferro; Espectroscopia Mössbauer; Fósforo; Alumínio.

\section{OCCURRENCE OF ALUMINUM AND PHOSPHORUS IN IRON ORES}

\begin{abstract}
It is well known the undesirable effect that impurities such as phosphorus and aluminum cause to the products and the reduction of iron ores and refining of steels. The presence of phosphorus in high amounts in the iron ores can make the steel brittle, favoring the appearance of cracks. In the present study eight samples of iron ores coming from Vale's mines were used. Sequential extractions were made to remove specific forms of phosphorus. The residues of these extractions, as well as the original samples, were characterized by X-ray diffraction, Mössbauer spectroscopy and chemical analyses. The results of the chemical extractions suggest that phosphorus and aluminum are associated to the iron oxides. For most samples there is a correlation between the presence of phosphorus and goethite $(\alpha-\mathrm{FeOOH})$, but some samples do not contain goethite but present high contents of phosphorus. The association of aluminum to the iron oxides suggests that it might not be possible to remove it by physical methods.
\end{abstract}

Key words: Iron ore; Mössbauer spectroscopy; Phosphorus; Aluminum.

\section{INTRODUÇÃO}

É bem conhecido o efeito indesejável que impurezas como o fósforo e alumínio causam aos produtos e operações de aglomeração e redução de minério de ferro e refino dos aços. A presença de fósforo em teores elevados torna o aço quebradiço e com fraturas, o que influencia o valor de mercado do minério de ferro. Em linhas gerais, o alumínio, na forma de óxidos, contribui para fragilização do sínter e altera a viscosidade das escórias de Alto-Forno, podendo comprometer a operação. Os métodos usuais de beneficiamento normalmente não são capazes de remover totalmente o fósforo presente em alguns tipos de minérios de ferro, a fim de tornar o teor deste contaminante aceitável abaixo de $0,05 \%$.(1) A forma na qual o fósforo e o alumínio se encontram nos minérios

\footnotetext{
'Estudante de Mestrado, Programa de Pós-gradução em Engenharia Mineral, Universidade Federal de Ouro Preto - UFOP. Campus Universitário Morro do Cruzeiro, s/n, Bauxita, Cep 35400-000, Ouro Preto, MG, Brasil. E-mail: mrclinha@yahoo.com.br ${ }^{2}$ Doutor, Departamento de Química, Universidade Federal de Ouro Preto - UFOP. Campus Universitário Morro do Cruzeiro, s/n, Bauxita, Cep 35400-000, Ouro Preto, MG, Brasil. E-mail: magela@iceb.ufop.br ${ }^{3}$ Estudante de Mestrado, Programa de Pós-gradução em Engenharia Mineral, Universidade Federal de Ouro Preto - UFOP. Campus Universitário Morro do Cruzeiro, s/n, Bauxita, Cep 35400-000, Ouro Preto, MG, Brasil. E-mail: aclaudiacarioca@yahoo.com.br ${ }^{4}$ M.Sc. Engenharia Metalúrgica, Centro de Tecnologia de Ferrosos - CTF, Vale. Estrada para o Morro do Chapéu, s/n, Cep 34000-000, Nova Lima, MG, Brasil. E-mail: flavio.dutra@vale.com
} 
de ferro ainda não é bem conhecida, mas com base em resultados encontrados para amostras sintéticas de goethita $(\alpha-\mathrm{FeOOH})$ e hematita $\left(\alpha-\mathrm{Fe}_{2} \mathrm{O}_{3}\right)$ pode-se inferir que o fósforo, provavelmente na forma de fosfato, se encontra adsorvido na superfície das partículas ou ocluído nos microporos. Outra possibilidade é que esteja localizado dentro da estrutura dos oxi-hidróxidos ou ainda como um mineral fosfatado. ${ }^{(2-4)}$ Para amostras sintéticas a literatura relata que a goethita apresenta maior capacidade de adsorção de fosfato que a hematita, certamente devido à maior área superficial e porosidade das partículas de goethita. ${ }^{(2)}$ Atualmente não existe nenhuma tecnologia que seja técnica e economicamente viável para a remoção de fósforo e alumínio no minério de ferro. A compreensão do modo de ocorrência desses elementos nos minérios certamente trará novas informações que, eventualmente, poderão subsidiar o desenvolvimento de métodos para a sua remoção. Neste trabalho são relatados os resultados de caracterização de fósforo e alumínio em oito amostras de minérios de ferro de diferentes minas e jazidas da Vale.

\section{MATERIAIS E MÉTODOS}

Foram investigadas oito amostras de minério de ferro provenientes de diferentes minas e jazidas da Vale. Análises químicas mostraram que, em geral, fósforo e alumínio estão mais concentrados nas faixas $>0,15 \mathrm{~mm}$ e $>0,045 \mathrm{~mm}$ sendo estas as frações escolhidas para estudo. As caracterizações química e mineralógica foram realizadas a partir de análises químicas via úmida e Inductively Coupled Plasma (ICP), difração de raios $X$ e espectroscopia Mössbauer. Extrações seqüenciais, baseadas nos métodos de Mehra e Jackson ${ }^{(5)}$ e Ruttenberg, ${ }^{(6)}$ foram realizadas em todas as amostras. $O$ método consiste em uma série de extrações sequenciais que extraem formas especificas de fósforo. Nas extrações com cloreto de magnésio (etapa I) e acetato de sódio (etapa II) supostamente ocorre a remoção de fósforo lábil e fósforo ligado a carbonatos e apatita, respectivamente. $\mathrm{Na}$ etapa III a extração é feita com o sistema ditionito-citrato-bicarbonato (DCB) e, em princípio, fósforo e alumínio ligados a óxidos de ferro são removidos. $O$ teor original de ferro das amostras foi determinado por titulação com dicromato de potássio, que é o método padrão recomendado e adotado na indústria. Devido à limitação de massa, os teores de ferro nos resíduos após extração foram feitos por ICP. O erro médio entre as análises químicas, por via úmida e por ICP, para as amostras originais, é de cerca de $1 \%$ absoluto, o que pode ser considerado aceitável para os propósitos deste trabalho. Os difratogramas de raios $X$ foram obtidos em um difratômetro Shimadzu XRD 6000, equipado com tubo de ferro e monocromador de grafite. As varreduras foram feitas entre 5 e $75^{\circ}(2 \theta)$ com velocidade de $1 \%$ min utilizando silício como padrão interno. As intensidades integradas e larguras dos picos foram obtidas pelo ajuste com uma função de Pearson após a subtração do ruído de fundo e do $\mathrm{K}_{2}{ }^{\left({ }^{(7)}\right.}$ Os espectros Mössbauer foram obtidos em um espectrômetro convencional operando à temperatura ambiente e ajustados computacionalmente com sextetos e/ou dubletos simétricos ou com distribuições de campos magnéticos hiperfinos. ${ }^{(8)}$

\section{RESULTADOS E DISCUSSÃO}

De acordo com os resultados das análises químicas, todas as amostras apresentam mais de $50 \%$ de ferro, exceto a amostra AM4, que apresentou uma concentração média de $36 \%$ (Tabela I). As amostras de maior concentração de alumínio são AM3 e AM5 com teores acima de $1,0 \%$ e de fósforo AM2, AM5 e AM8, com teores acima de 0,11\%.

Tabela I. Análises químicas (\% p/p) das amostras originais de granulometria $>0,150 \mathrm{~mm}$ e $>0,045 \mathrm{~mm}$

\begin{tabular}{cccc}
\hline Amostra & Fe & Al & P \\
\hline AMI-I50 & 63,8 & 0,198 & 0,062 \\
AMI-45 & 65,0 & 0,171 & 0,058 \\
AM2-I50 & 68,5 & 0,673 & 0,118 \\
AM2-45 & 68,1 & 0,314 & 0,114 \\
AM3-I50 & 56,8 & 1,366 & 0,049 \\
AM3-45 & 56,7 & 1,264 & 0,055 \\
AM4-150 & 36,0 & 0,046 & 0,032 \\
AM4-45 & 36,4 & 0,090 & 0,055 \\
AM5-150 & 61,9 & 1,143 & 0,127 \\
AM5-45 & 62,0 & 0,957 & 0,119 \\
AM6-250 & 64,7 & 0,676 & 0,057 \\
AM7-I50 & 68,1 & 0,177 & 0,071 \\
AM7-45 & 67,7 & 0,391 & 0,087 \\
AM8-I50 & 64,6 & 0,117 & 0,169 \\
AM8-45 & 57,5 & 0,101 & 0,129 \\
\hline
\end{tabular}

A mineralogia das amostras consiste basicamente de hematita, goethita, magnetita e quartzo. Em várias amostras a goethita apresenta tamanhos médios de cristalito inferiores a $100 \mathrm{~nm}$, sendo que na amostra AM3 o tamanho varia de $20 \mathrm{~nm}$ a $30 \mathrm{~nm}$. Para a hematita os tamanhos normalmente são acima de $100 \mathrm{~nm}$, exceto para a amostra 5 , que apresentou tamanho médio de $60 \mathrm{~nm}$.

Nas etapas I e II da extração sequencial não foi observada remoção significativa de fósforo e alumínio, mostrando, portanto, que não existem teores elevados de fósforo lábil ou ligado a carbonatos nas amostras. Na etapa III foram realizadas duas extrações sucessivas com DCB, e os percentuais de remoção de fósforo e alumínio foram os mais significativos dentre as demais etapas. Utilizaram-se duas extrações com DCB devido à maior resistência à dissolução dos óxidos de ferro com alta cristalinidade ou elevada substituição de $\mathrm{Fe}^{3+}$ por $\mathrm{Al}^{3+}$ na estrutura. ${ }^{(9)}$ Observa-se que para a maioria das amostras ocorre extração significativa do fósforo e do alumínio nesta etapa. Os espectros Mössbauer das amostras $>0,045 \mathrm{~mm}$ antes e após a extração com DCB estão mostrados nas Figuras I, 2 e 3 . A partir das áreas relativas obtidas dos ajustes destes espectros e dos resultados das análises químicas dos resíduos calculou-se a mineralogia das amostras. É possível observar com os resultados numéricos da 
Tabela 2 que, para a maior parte das amostras, o tratamento com DCB removeu preferencialmente a goethita. É interessante observar que a amostra AM2 praticamente não apresenta goethita, mas contém $0,12 \%$ de fósforo. Esta observação mostra que o fósforo pode não estar necessariamente associado à goethita.

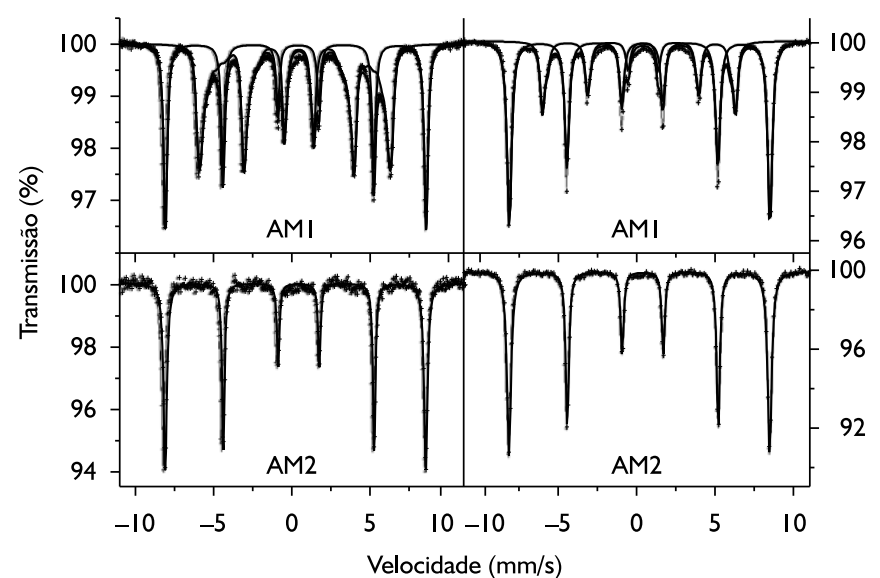

Figura I. Espectros Mössbauer das amostras AMI e AM2 originais (à esquerda) e após extração com DCB (à direita).

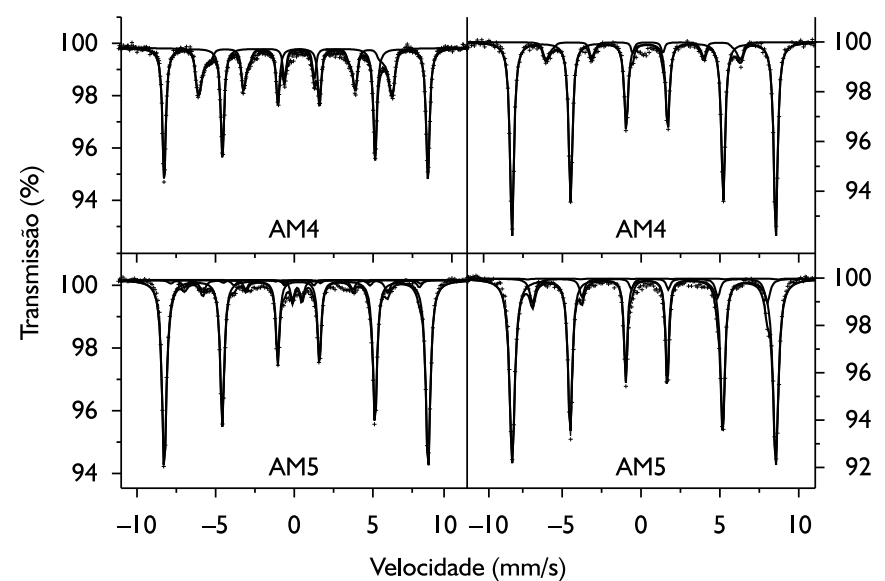

Figura 2. Espectros Mössbauer das amostras AM4 e AM5 originais (à esquerda) e após extração com DCB (à direita).

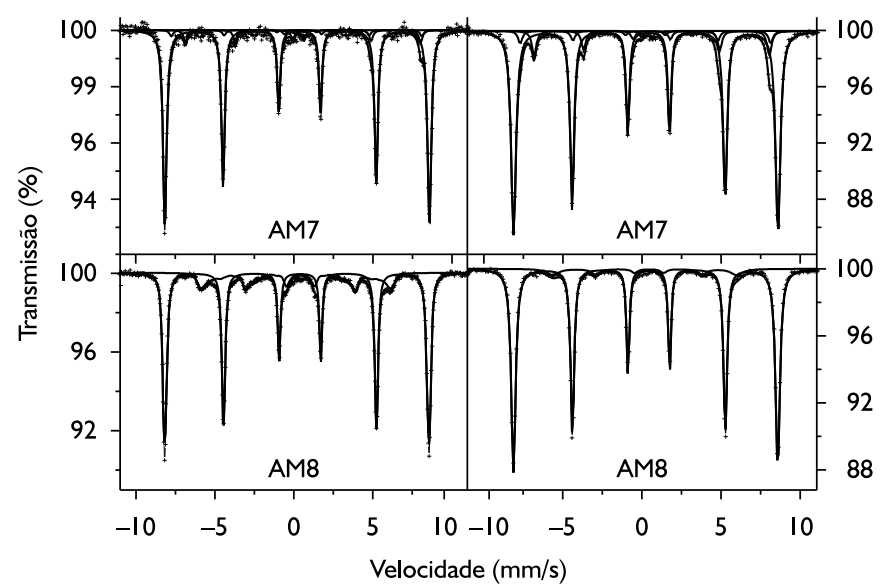

Figura 3. Espectros Mössbauer das amostras $A M 7$ e $A M 8$ originais (à esquerda) e após extração com DCB (à direita).
Tabela 2. Teores $(\% \mathrm{p} / \mathrm{p})$ de hematita $(H)$, goethita $(\mathrm{G})$, magnetita $(\mathrm{Mt})$ e quartzo $(\mathrm{Qz})$ antes e após a etapa III da extração sequencial

\begin{tabular}{|c|c|c|c|c|c|c|c|c|}
\hline \multirow[t]{2}{*}{ Amostras } & \multicolumn{4}{|c|}{ Antes } & \multicolumn{4}{|c|}{ Após } \\
\hline & $\mathbf{H}$ & G & Mt & Qz & H & G & Mt & Qz \\
\hline AMI-45 & 36 & 63 & - & 1 & 63 & 31 & - & 6 \\
\hline AM2-45 & 97 & - & - & 3 & 97 & - & - & 3 \\
\hline AM3-45 & 2 & 88 & - & 10 & I & 89 & - & 10 \\
\hline AM4-45 & 33 & 21 & - & 46 & 24 & 3 & - & 73 \\
\hline AM5-45 & 74 & 10 & 6 & 10 & 79 & 0 & 10 & II \\
\hline AM6-250 & 93 & - & - & 7 & 93 & - & - & 7 \\
\hline AM7-45 & 86 & 2 & 8 & 4 & 81 & I & 13 & 5 \\
\hline AM8-45 & 64 & 20 & - & 16 & 67 & 6 & - & 27 \\
\hline
\end{tabular}

Uma questão importante do ponto de vista industrial está relacionada à possível remoção de alumínio durante as várias etapas de concentração do minério de ferro. Além de estar presente em óxidos e hidróxidos de alumínio ou em silicatos, existe a possibilidade de o alumínio substituir isomorficamente o ferro na estrutura da hematita e/ ou da goethita. Os limites máximos de substituição para amostras naturais de hematita e goethita são, respectivamente, $15 \mathrm{~mol} \%$ e $33 \mathrm{~mol} \%$. $^{(10)}$ Estes valores correspondem a teores de alumínio de $5,4 \%$ e $10,2 \%$ em peso na hematita e na goethita, respectivamente. Portanto, os teores encontrados nas presentes amostras (Tabela I) estão dentro desta faixa de solubilidade, sugerindo que todo alumínio pode estar na estrutura dos óxidos de ferro. Por outro lado, as extrações com DCB fornecem informações mais precisas a respeito dos teores de alumínio existentes nos minerais de ferro visto que este tratamento remove seletivamente apenas os óxidos de ferro. ${ }^{(5)}$ Supondo-se que $\circ$ alumínio esteja apenas na estrutura da hematita nas amostras AM2 e AM6, e na estrutura da goethita nas demais amostras, podem-se calcular os valores de $x$ nas fórmulas $\left(\mathrm{Fe}_{1-x} \mathrm{Al}_{x}\right)_{2} \mathrm{O}_{3}$ e $\left(\mathrm{Fe}_{1-\mathrm{x}} \mathrm{Al}_{\mathrm{x}} \mathrm{OOH}\right)$, a partir dos teores das amostras originais e os existentes nos resíduos da etapa III da extração sequencial (Tabela 3). É facilmente encontrado que $x$ varia entre $0,1 \mathrm{~mol} \%$ até o máximo de 2,5 mol\%.

Para a maioria das amostras existe uma correlação entre a extração de alumínio e de fósforo, sugerindo que ambos os elementos estão associados aos óxidos de ferro, provavelmente à goethita. Entretanto, as amostras AM2 e AM6 não possuem goethita e apresentaram um percentual de remoção elevado. Isto pode ser devido à presença de óxidos de ferro litogênicos como a magnetita e hematita, ${ }^{(11)}$ que, embora sejam considerados resistentes ao procedimento $\mathrm{DCB}_{80}$, podem solubilizar pequenas quantidades de ferro em cada extração sucessiva. Por outro lado, a amostra AM8 possui $20 \%$ de goethita e 
Tabela 3. Teores de alumínio e de fósforo (\%) antes e após a etapa III da extração sequencial

\begin{tabular}{ccccccc}
\hline Amostra & Al original & Al residual & $\mathbf{A l}_{\mathrm{Fe}}$ & $\mathbf{P}_{\text {original }}$ & $\mathbf{P}$ residual & $\mathbf{P}_{\mathrm{Fe}}$ \\
\hline AMI-45 & $0,17 \mathrm{I}$ & 0,033 & $8 \mathrm{I}$ & 0,058 & 0,018 & 68 \\
AM2-45 & 0,314 & 0,102 & 68 & 0,114 & 0,038 & 67 \\
AM3-45* & $\mathrm{I}, 264$ & - & - & 0,055 & - & - \\
AM4-45 & 0,090 & 0,006 & 93 & 0,055 & 0,009 & 84 \\
AM5-45 & 0,957 & $0,14 \mathrm{I}$ & 85 & 0,119 & 0,035 & 75 \\
AM6-250 & 0,676 & 0,374 & 45 & 0,057 & $0,04 \mathrm{I}$ & 28 \\
AM7-45 & $0,39 \mathrm{I}$ & 0,057 & 85 & 0,087 & 0,015 & 83 \\
AM8-45 & $0,10 \mathrm{I}$ & 0,070 & $3 \mathrm{I}$ & 0,129 & 0,023 & 82 \\
\hline
\end{tabular}

* A massa da amostra após a extração foi insuficiente para a realização da análise química.

apresentou uma extração de cerca de $30 \%$. Os diferentes teores de martita e especularita certamente têm grande influência na quantidade de hematita que é extraída pelo $D C B$, mas este efeito ainda não está claro. Os teores de $\mathrm{Al}_{\mathrm{Fe}}$ e de $\mathrm{P}_{\mathrm{Fe}}$ na Tabela 3 significam os percentuais de alumínio e de fósforo associados aos óxidos de ferro em relação aos teores das amostras originais. Considerando-se que $\circ$ tratamento com DCB não removeu completamente os óxidos de ferro (Tabela 2), é possível que o alumínio existente nos resíduos também esteja substituindo isomorficamente o ferro. Em conclusão, um percentual significativo do alumínio e fósforo existente nessas amostras não poderá ser removido por métodos físicos de concentração.

\section{CONCLUSÕES}

Os resultados das extrações sequenciais sugerem que grande proporção do total de alumínio está substituindo isomorficamente o ferro na estrutura dos óxidos de ferro, mais precisamente na goethita. $O$ fósforo também parece estar associado aos óxidos de ferro, e foi removido simultaneamente com o alumínio, exceto nas amostras AM6 e AM8. Os espectros Mössbauer apresentam uma redução superior a $50 \%$ nos teores de goethita de algumas amostras após extração com DCB. Como o teor de fósforo também diminuiu, é razoável inferir que este elemento está associado diretamente à goethita. No entanto, é possível concluir que uma significativa porcentagem de alumínio e fósforo não pode ser removida destas amostras por métodos físicos de concentração.

\section{Agradecimentos}

Este trabalho foi parcialmente financiado pela Vale, CNPq e Fapemig.

\section{REFERÊNCIAS}

I CHENG, C. Y. et al. Dephosphorisation of Western Australian iron ore by hydrometallurgical process. Minerals Engineering, v. 12, n. 9, p. 1083-92, May 1999.

2 COLOMBO, C.; BARRÓN, V.; TORRENT, J. Phosphate adsorption and desorption in relation to morphology and crystal properties of synthetic hematites. Geochimica et Cosmochimica Acta, v. 58, n. 4, p. I26I-9, Oct. 1994.

3 GÁLVEZ, N.; BARRÓN, V.; TORRENT, J. Preparation and properties of hematite with structural phosphorus. Clays and Clay Minerals, v. 47, n. 3, p. 375-85, Feb. 1999.

4 TORRENT, J.; SCHWERTMANN, U.; BARRÓN, V. Fast and slow phosphate sorption by goethite-rich natural materials. Clays and Clay Minerals, v. 40, n. I, p. I4-2I, 1992.

5 MEHRA, O. P.; JACKSON, M. L. Iron oxide removal from soils by a dithionite-citrate system buffered with sodium bicarbonate. In: NATIONAL CLAY CONFERENCE, 7., 1960. Proceedings... New York: Pergamon Press, 1960. p. 317-27.

6 RUTTENBERG, K. C. Development of a sequential extraction method for different forms of phosphorus in marine sediments. Limnology and Oceanography, v. 37, n. 7, p. 1460-82, June 1992.

7 JENKINS, R.; SNYDER, R. L. Introduction to X-Ray powder diffractometry. New York: John Wiley \& Sons, 1996.

8 WIVEL, C.; MORUP, S. Improved computational procedure for evaluation of overlapping hyperfine parameter distributions in Mössbauer spectra. Journal of Physics, v. EI4, n. 5, p. 605-10, May 198I.

9 INDA JUNIOR, A. V.; KÄMPF, N. Avaliação de procedimentos de extração dos óxidos de ferro pedogênicos com ditionito-citrato-bicarbonato de sódio. Revista Brasileira de Ciências do Solo, v. 27, p. I I 39-47, Nov./Dec. 2003.

IO STANJEK, H.; SHWERTMANN, U. The influence of aluminum on iron oxides. Part XVI: Hidroxyl and aluminum substitution in synthetic hematites. Clays and Clay Minerals, v. 40, n. 3, p. 347-54, May 1992.

II RESENDE, M. Mineralogy, chemistry, morphology and geomorphology of some soils of the Central Plateau of Brazil. In: apud INDA JUNIOR, A. V.; KÄMPF, N. Avaliação de procedimentos de extração dos óxidos de ferro pedogênicos com ditionito-citrato-bicarbonato de sódio. Revista Brasileira de Ciências do Solo, v. 27, n. 6, p.l139-47, nov./dez. 2003.

Recebido em: 13/04/2009

Aceito em: 30/06/2010 